\title{
Positive/Negative Phototropism: Controllable Molecular Actuators with Different Bending
}

\section{Behavior}

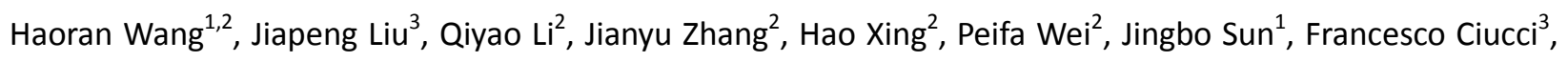
Jacky W. Y. Lam², Ran Lư $^{1^{*}}$, Ben Zhong Tang ${ }^{2 *}$

${ }^{1}$ State Key Laboratory of Supramolecular Structure and Materials, College of Chemistry, Jilin University, Changchun, China.

${ }^{2}$ Department of Chemistry, Hong Kong Branch of Chinese National Engineering Research Center for Tissue Restoration and Reconstruction and Institute for Advanced Study, The Hong Kong University of Science and Technology, Hong Kong, China.

${ }^{3}$ Department of Mechanical and Aerospace Engineering, The Hong Kong University of Science and Technology, Hong Kong, China.

*Corresponding Authors: luran@mail.jlu.edu.cn; tangbenz@ust.hk

\begin{abstract}
Herein, a series of molecular actuators based on the crystals of (E)-2-(4-fluorostyryl)benzo[d]oxazole (BOAF4), (E)-2-(2,4-difluorostyryl)benzo[d]oxazole (BOAF24), (E)-2-(4-fluorostyryl)benzo[d]thiazole (BTAF4) and (E)-2(2,4-difluorostyryl)benzo[d]thiazole (BTAF24) showed unprecedented different bending behavior under UV irradiation. BOAF4 and BTAF4 bent towards light, whereas BOAF24 and BTAF24 bent away from light. Although the chemical structures of these compounds are similar, we found out the $\mathrm{F} \cdots \mathrm{H}-\mathrm{C}$ interaction was the main driving force for the different molecular packing in the crystals, which led to the positive/negative phototropism of the actuators. Moreover, the theoretical calculation was carried out to reveal the mechanical properties of the crystals. Taking advantage of the photo responsive property, we achieved the potential application in pushing objects, as well as enriching and removing pollutants. This system not only achieved a class of molecular actuators with different bending behavior through introducing different number of $F$ atom, but also realized pushing and catching behavior within one molecule, which opens a novel gate for crystal engineering.
\end{abstract}




\section{Keywords}

crystal engineering, actuators, phototropism, [2+2] cycloaddition, photomechanical effects

\section{Introduction}

Nature endows organisms with tropisms for moving toward or away from a stimulus. For most plants, their stem exhibit positive phototropism to maximize photosynthetic energy for promoting growth. ${ }^{1,2}$ However, most roots and some vine shoot tips exhibit negative phototropism, allowing them to grow towards darkness, drill into soil and climb objects. ${ }^{3}$ The combination of positive and negative phototropism allows plants to grow in the correct direction, in order to make better use of light energy. Inspired by the response of natural organisms towards external stimuli, various artificial intelligent materials, such as artificial muscles and flexible electronics, have been explored and exploited. ${ }^{4-10}$ Among them, photomechanical responsive materials have emerged as a research hotspot, with unique advantages including easy to achieve contactless remote control and realize the miniaturization of devices. ${ }^{11,12}$ Especially, photomechanical molecular crystals exhibit some conspicuous merits over polymer-based materials, such as faster response time, ${ }^{13}$ higher Young's modulus, ${ }^{14}$ and an ordered crystal structure which can be easily characterized by X-ray diffraction techniques. ${ }^{6}$ Additionaly, an ordered crystal structure offers chances for rapid energy transfer between tightly packed molecules, and this could be utilized as a physical platform for actuation from photochemical reaction

from nanoscale to macroscale. ${ }^{15,16}$ It has been found that the crystals based on photochemical active organic compounds, including azobenzenes, anthracenes, diarylethenes and furylfulgides, are capable of bending, ${ }^{17-29}$ curling, ${ }^{30}$ twisting, ${ }^{31,32}$ crawling, $^{33-37}$ and leaping ${ }^{5,38}$ in response to light.

Recently, a kind of crystal which could reversibly respond to multiple external stimuli (heat, UV light, and mechanical pressure) by twisting, bending, and elastic deformation without fracture was reported by Naumov et al. ${ }^{39}$ And Bardeen et al. reported the controllable movement of molecular crystals based on 1,2-bis(2methyl-5-phenyl-3-thienyl)perfluorocyclopentene via varying the angle of incident light. ${ }^{40}$ As far as we know, 
in order to regulate crystal motion behavior, previous work mainly focused on changing external conditions e.g. controlling the crystal habits and the irradiation direction. Thus, it is highly challenging but rewarding to realize varing motion modes such as positive and negative phototropism through crystal structure arrangement regulation.

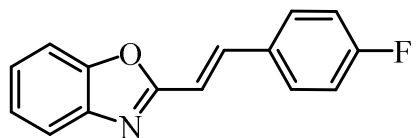

BOAF4

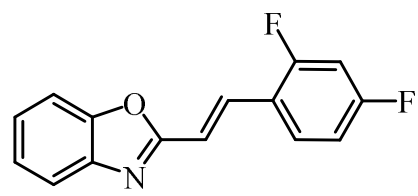

BOAF24

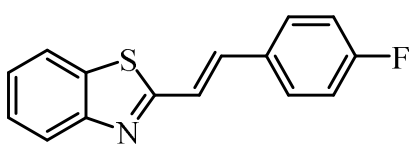

BTAF4

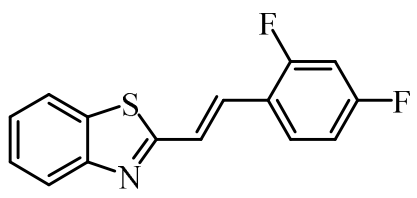

BTAF24

Scheme 1 Molecular structures of fluoro-containing styrylbenzoxazoles and styrylebenthiazoles.

In previous work, we found that the introduction of chlorine at different position of benzene in styrylbenzoxazoles could affect the molecular packing in the crystals, which would further affect the topophotochemical reaction. ${ }^{41}$ Compared with chlorine, fluorine possesses a smaller atomic radius and a stronger electronegativity, so introducing fluorine into conjugated organic molecules might lead to unique arrangement in crystals and interesting photomechanical behaviors. With these in mind, we intend to study the photo-induced mechanical motions of the molecular crystals based on fluoro-containing styrylbenzoxazoles (BOAF4 and BOAF24) and styrylbenzothiazoles (BTAF4 and BTAF24, Scheme 1) so as to reveal the effect of the molecular structures and crystal habits on the photomechanical behavior. It was found that the crystals of BOAF4, BOAF24, BTAF4 and BTAF24 with different habits showed photo-induced bending, curling, fragment, swelling and photosalient behavior. Interestingly, under the UV irradiation, the crystals of BOAF4 and BTAF4 showed photo-induced bending towards light, but the crystals of BTAF4 and BTAF24 showed opposite photo-induced bending away from light. 


\section{Experimental Methods}

All Experimental Methods are included in the Supporting Information.

\section{Results and Discussion}

\section{Photomechanical behavior}

Firstly, the slice-like crystals and rod-like crystals of BOAF4 were obtained from petroleum ether $/ \mathrm{CH}_{2} \mathrm{Cl} 2 \mathrm{v} / \mathrm{v}=$ $3 / 1$ ) in one test tube. In the case of the slice-like crystal with low aspect ratio, the photo-induced swinging and swelling were observed in Supporting Information Figure S1 and Video S1. Comparatively, the thinner slice-like crystal displayed a different behavior that it could bent towards UV light (Supporting Information Video S2 and Figure S2). Interestingly, for irregular rod-like crystal of BOAF4 with high aspect ratio, comparable bending behavior was observed under UV irradiation. Besides exhibiting light-induced swelling, it could roll up its tail and show bending towards the irradiated direction under UV light (Figure 1 and Supporting Information Video S3). In detail, the UV irradiation induced the emergence of cracks in the backlight side of the crystal, and the tail bent towards light. When the irradiation time was prolonged to $90 \mathrm{~s}$, the tail was raised out of the plane, and the bending angle was ca. $135^{\circ}$ without break.

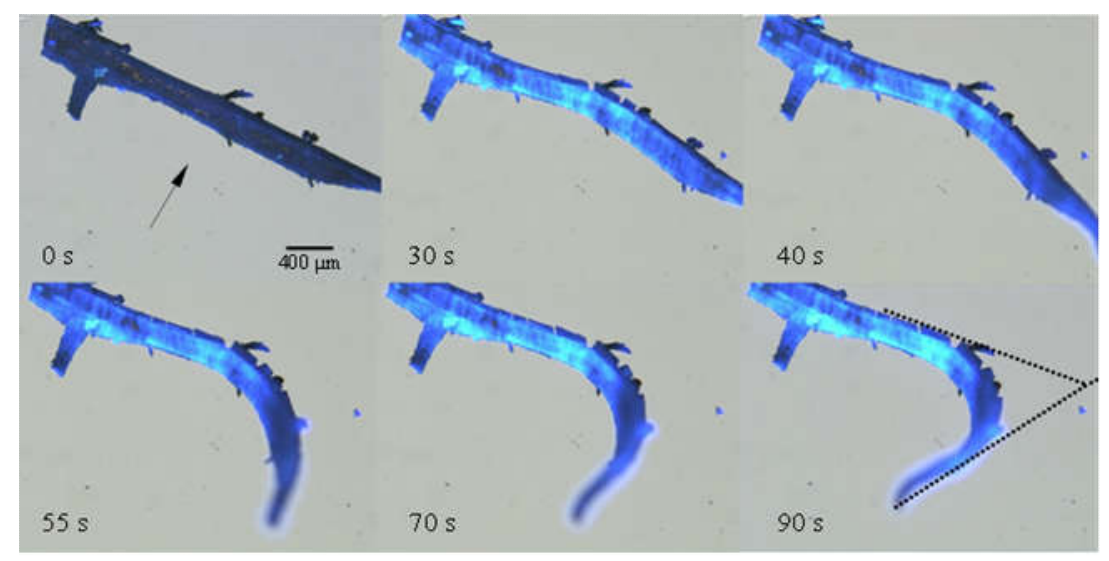

Figure 1 Optical photos of the irregular rod-like crystal of BOAF4 before and after irradiation vertically with $365 \mathrm{~nm}$ light for different time (the arrow indicates the irradiation direction). 
Therefore, BOAF4 crystals with smaller thickness or high aspect ratio tend to bend towards light source. Notably, in our previous work, the needle-like crystals of (E)-2-(2,4-dichlorostyryl)benzo[d]oxazole (BOACl24) bended away from the UV light, ${ }^{41}$ opposite to the rod-like crystals of BOAF4. To further research the positive and negative phototropism behavior of crystals under UV irradiation, BOAF24 was designed and synthesized to compare with BOAF4. ${ }^{42}$

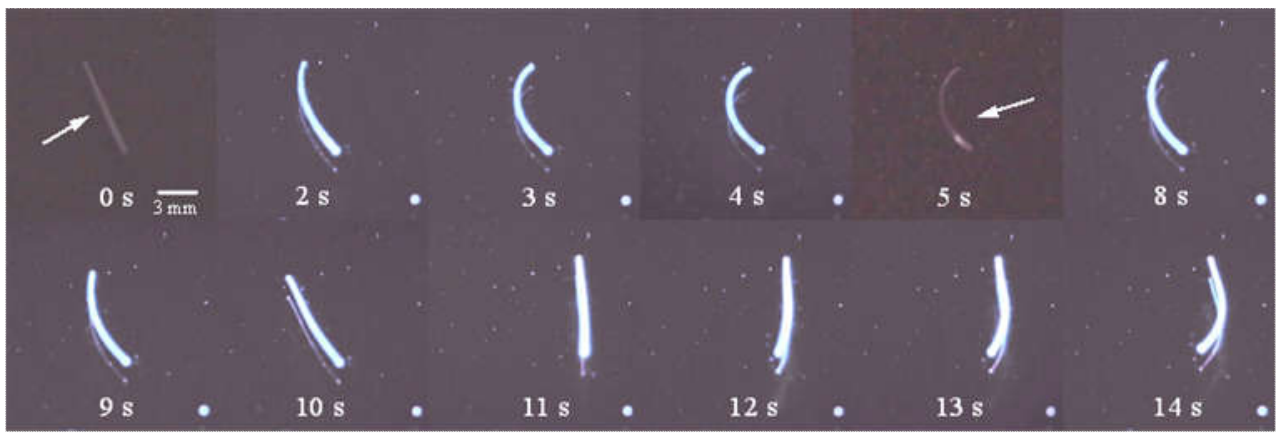

Figure 2 Optical photos of rod-like crystals of BOAF24 before and after irradiation with $365 \mathrm{~nm}$ light for different time (the arrows indicate the irradiation direction).

BOAF24 formed block, ribbon-like and rod-like crystals in petroleum ether $/ \mathrm{CH}_{2} \mathrm{Cl}_{2}(\mathrm{v} / \mathrm{v}=3 / 1)$. For the long block irregular crystal of BOAF24, it could swing upon UV irradiation, and small pieces jumped away from the body. When prolonging the UV irradiation, more cracks were observed in the cross sections of the crystal (Supporting Information Video S4). Besides, we visualized the energy accumulation state in the photomechanical process which P. Naumov et al. proposed. ${ }^{6}$ As shown in Supporting Information Video S5, after we turned off the UV lamp at $23 \mathrm{~s}$, the crystal of BOAF24 laid still at that time but began to break after the subsequent $1 \mathrm{~s}$. It proves that the photosalient occurs after an induction period during which energy accumulates. Further, we found that 1D crystal bent away from irradiation and the thinner crystals marked exhibited more significant bending compared with the thicker ones (Supporting Information Video S6 and Figure S3a). When the crystals were vertically irradiated by UV light from downside, the right side of the elongated crystal marked in red circle bent up and could be bent down via turning around the irradiation 
direction. Meanwhile, the crystal part marked in yellow circle gave a slight movement since it was thicker. Also, the bouquet-like crystals consisting lots of ribbons displayed similar behavior (Supporting Information Figure S3b). Except for the bending behavior, bouquet of ribbon-like crystals of BOAF24 (Supporting Information Video S7) could even jumped under UV irradiation. For clearly observation of the bending direction of the crystals, one rod-like crystal of BOAF24 was selected. As shown in Supporting Information Video S8 and Figure 2 , it bent away from light source obviously upon irradiated vertically by $365 \mathrm{~nm}$ light. When the irradiation direction was turned around, the bent crystal was then straightened. Prolonging the irradiation time, the crystal jumped at $11 \mathrm{~s}$ and continued to bend backwards UV light. Such behavior on bending away from light source could be repeated for several times, meaning that the light-induced bending process was reversible.
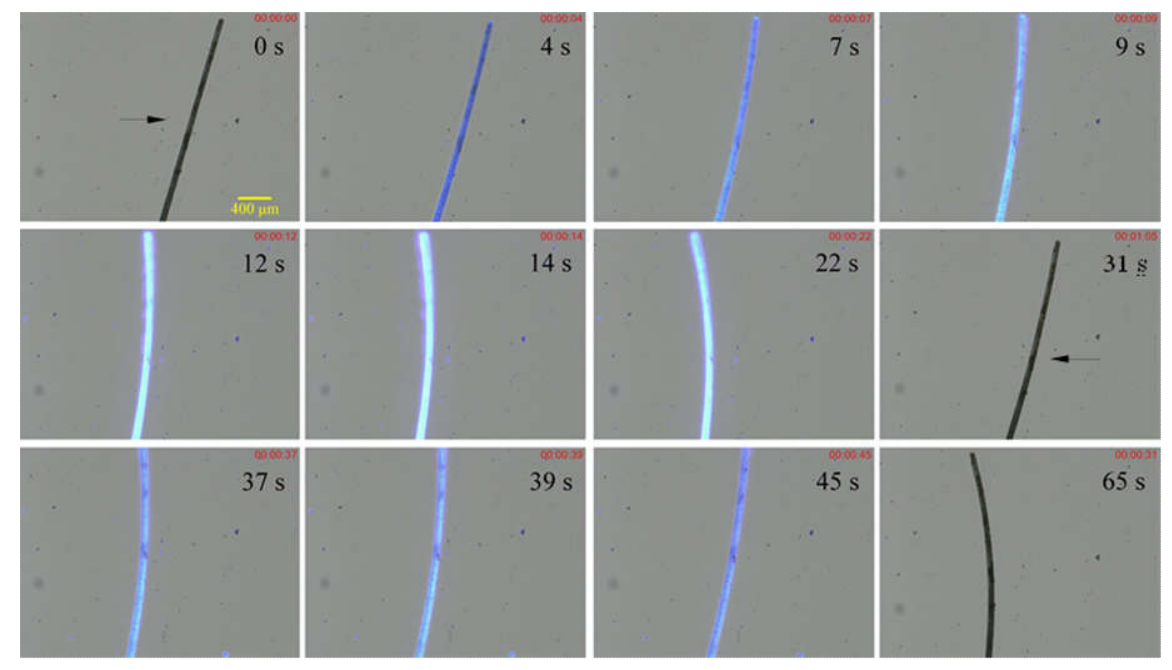

Figure 3 Optical images of the rod-like crystals of BTAF4 before and after irradiation with $365 \mathrm{~nm}$ light for different time (the arrows indicate the irradiation directions).

For deep understanding of the different photomechanical effects of BOAF4 and BOAF24, crystals of their analogues BTAF4 and BTAF24 were gained. As anticipated, a rod-like crystal of BTAF4 bent towards UV light, and the bent crystal could be straightened when the irradiation direction was changed to right side (Figure 3). Meanwhile, an enhanced fluorescence intensity was observed (Supporting Information Figure S4). ${ }^{43,44}$ Like 
BOAF4, the photoreaction product of BTAF4 is much probably AIE active. ${ }^{45}$ We also obtained the rod-like, block and slice-like crystals of BTAF24 from cyclohexane $/ \mathrm{CH}_{2} \mathrm{Cl}_{2}(\mathrm{v} / \mathrm{v}=4 / 1)$. Under UV irradiation, it was found that rod-like and slice-like crystals bent away from light source, whereas block crystals just jumped without any bending performance. As shown in Figure S5, after the irradiation with $365 \mathrm{~nm}$ light from upside vertically, the straight rod-like crystal bent backwards light source. When the irradiation direction changed to downside, the bent crystal could be straightened and further bent upward with prolonging the irradiation time. The behavior of bending backwards UV light could be repeated several times (Supporting Information Video S9). Furthermore, the block crystals of BTAF24 could jump away from its original positions when the UV irradiation time was $3 \mathrm{~s}$, and small pieces exploded out of the body (Supporting Information Video S10). The slice-like crystal of BTAF24 bent backwards light source in the beginning of the irradiation, followed by spitting into several parts perpendicular to the long axis of the crystal (Supporting Information Video S11).

\section{Mechanism study}

In our previous work, it was found that photo-induced [2+2] cycloaddition reaction took place in the molecular crystals of BOAF4 and BOAF24, which was the driving forces for the photomechanical effects. Under the UV irradiation, the bulk crystal of BOAF4 was "turned on" (from non-emissive to emissive) with crystal jump and broken. ${ }^{46}$ Moreover, ${ }^{1}$ H NMR spectral changes of BTAF24 before and after UV irradiation for different time suggested that the photodimerization occurred in the microcrystals. As depicted in Supporting Information Figure S7 after the microcrystals of BTAF24 were irradiated by UV light for 2 min, a double at 5.20 ppm emerged, which could be ascribed to the protons in newly formed cyclobutane. Meanwhile, new peak at $6.93 \mathrm{ppm}, 7.11 \mathrm{ppm}$ and $7.60 \mathrm{ppm}$ ascribed to the protons in the photodimerization product (named as DBTAF24)were also detected. Increasing the irradiation time resulted in the decrease of the integral values for the signals of BTAF24 and the increase of those of D-BTAF24. The similar ${ }^{1} \mathrm{H}$ NMR spectral changes of BTAF4 were observed during the UV irradiation of the microcrystals (Supporting Information Figures S8). It 
suggested that the photodimerization was also the driving force for the transformation from light into mechanical energy in crystals of BTAF4 and BTAF24.

To further demonstrate the driving force of the photomechanical behavior of the crystals, we collected the single crystal data of BOAF4, BOAF24, BTAF4 and BTAF24 as well as their dimers of D-BOAF4, D-BOAF24 ( $\alpha$ and $\beta$-types), D-BTAF4 and D-BTAF24. Among them, the single crystal structure of BOAF4, BOAF24 and DBOAF4, D-BOAF24 ( $\alpha$ - and $\beta$-types)had been reported in our previous work. ${ }^{45} \mathrm{G}$. Schmidt et al. found that the distance of "olefin pair" (less than $4.2 \AA$ ) as well as the angles of $\vartheta_{2}$ and $\vartheta_{3}$ (close to $90^{\circ}$ ) depicted in Supporting Information Chart S1 were considered to be geometric criteria for photodimerization. ${ }^{47}$ In the single crystal of BOAF4, the distance in "olefin pair" were $3.896 \AA$ and $3.681 \AA$ (Supporting Information Figure S9a), and $\vartheta_{2}$ and $\vartheta_{3}$ were ca. $77.00^{\circ} / 69.99^{\circ}$ and $87.97^{\circ} / 83.04^{\circ}$, respectively (Supporting Information Table S3). Therefore, such molecular packing made the photodimerization of BOAF4 accessible. Subsequently, single crystal structure of D-BOAF4 was analyzed for further investigation. For D-BOAF4, two isomers (isomer I and II) were achieved via photocycloaddition. Take isomer I for instance, the bond lengths of the newly formed C-C bond in the four-membered ring were $1.578 \AA$ and $1.579 \AA$. Therefore, each carbon atom in the original $\mathrm{C}=\mathrm{C}$ had to move ca. 1.1 1.3 ̊̊ during photodimerization. Meanwhile, the newly formed cyclobutane impelled the benzoxazole and benzene to move away from the four-membered ring, like a bird spreads its wings. As a result, the distances between the outermost $\mathrm{F}$ and $\mathrm{C}$ atoms reached $7.132 \AA$ and $7.811 \AA$. For single crystal structures of BOAF24, BTAF4 and BTAF24, similar results were found (Supporting Information Figure S9b, S10 and S11). Subsequently, comparing the molecular volume in the single crystal structure before and after photocycloaddition gave us an inspiration. Upon dimerization, the molecular volume was amplified and then strain was yielded, leading to the mechanical movements of crystals.

It is time to discuss why BOAF4 and BTAF4 bent towards UV light, while BOAF24 and BTAF24 exhibited oppositee backlight bending performance. Luckily, we got some inspiration from azobenzene mesogens. In 2003, Ikeda and coworkers found that monodomain and polydomain liquid crystal elastomers (LCE) films 
showed different light induced bending behavior: the bending of the former took place just along the alignment direction of the azobenzene mesogens. ${ }^{48,49}$ And in 2006, they found that the initial alignment of photoactive mesogens significantly affected the bending behavior of the LCE films: the homogeneous films bent toward the irradiation direction of the actinic UV light, while the homeotropic films bent away from the light source. ${ }^{50}$ Thus, we proposed that the arrangement of molecules in the crystal have a crucial impact on the bending behavior.

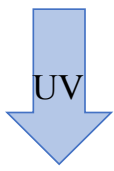

Before

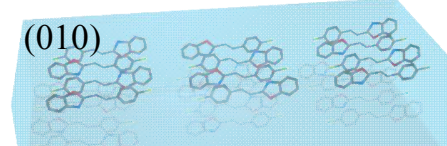

After

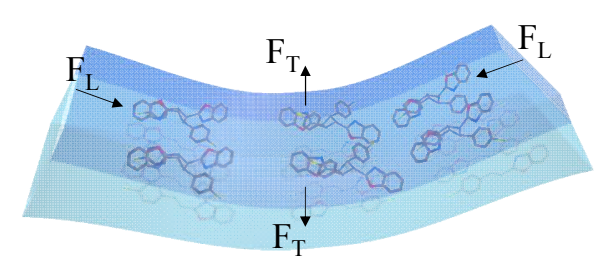

$\mathrm{b}$
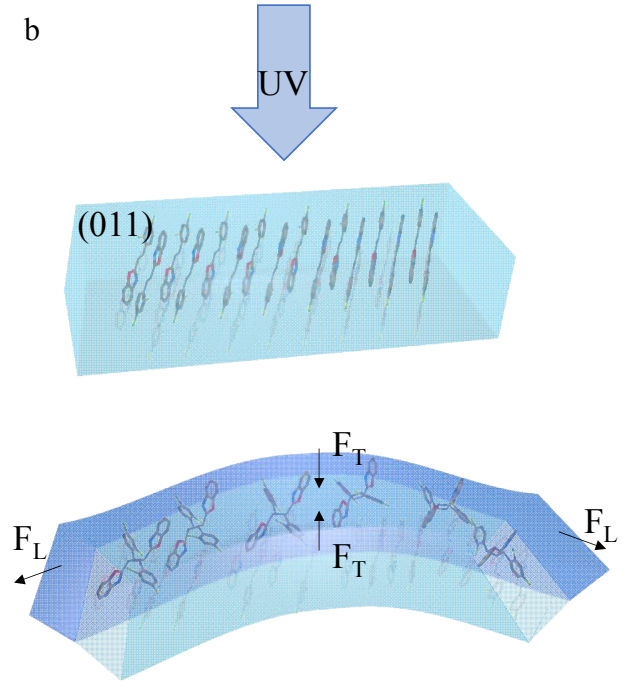

Figure 4 Schematic illustration of the bending of BOAF4 (a)and BOAF24 (b) under UV irradiation.

Firstly, it is proved that strain would be generated from the phototropic surface of the crystal under the UV

irradiation. ${ }^{51}$ As shown in Figure 4a, the [2+2] cycloaddition mainly occurred on the (010) face of the crystal of BOAF4, which was the largest superficial area. Before UV irradiation, the molecules were longitudinally aligned along the long axis of the crystal. The molecular length decreased from $12.268 \AA$ (BOAF4)to ca. 11.091 $\AA$ (the average value of the two isomers of (D-BOAF4) upon photodimerization. However, the molecular width of D-BOAF4 was increased from $3.56 \AA$ (the average value of the distance of two BOAF4 molecules) to ca. $7.476 \AA$. So, on the (010) face of BOAF4 crystal, the force along longitudinal direction $\left(F_{L}\right)$ makes the crystal shrink, however the force along transverse direction $\left(\mathrm{F}_{\mathrm{T}}\right)$ leads to the expanding of crystal. Therefore, on (101) 
face the crystal was extended along the transverse direction, while $F_{L}$ makes it compressed along the longitudinal direction. As a result, the crystals of BOAF4 exhibited phototropic bending behavior. Similarly, the [2+2] cycloaddition reaction of BOAF24 took place on (011) face of the crystal, leading to the decrease of the molecular length from $12.286 \AA$ to $11.046 \AA$ (the average value of $\alpha$-type D-BOAF24) and the increase of the molecular width from average $3.863 \AA$ to $6.822 \AA$ (The average width of $\alpha$-type D-BOAF24). However, the molecular alignment of BOAF24 was different from that of BOAF4 in crystal. Molecules of BOAF24 aligned perpendicularly to the long axis of the crystal (Figure 4b). On (011) face, the crystal was compressed along the transverse direction and extended along the longitudinal direction, exhibiting backlight bending behavior. Similar observations were also found for BTAF4 and BTAF24.

\section{Theoretical calculations}

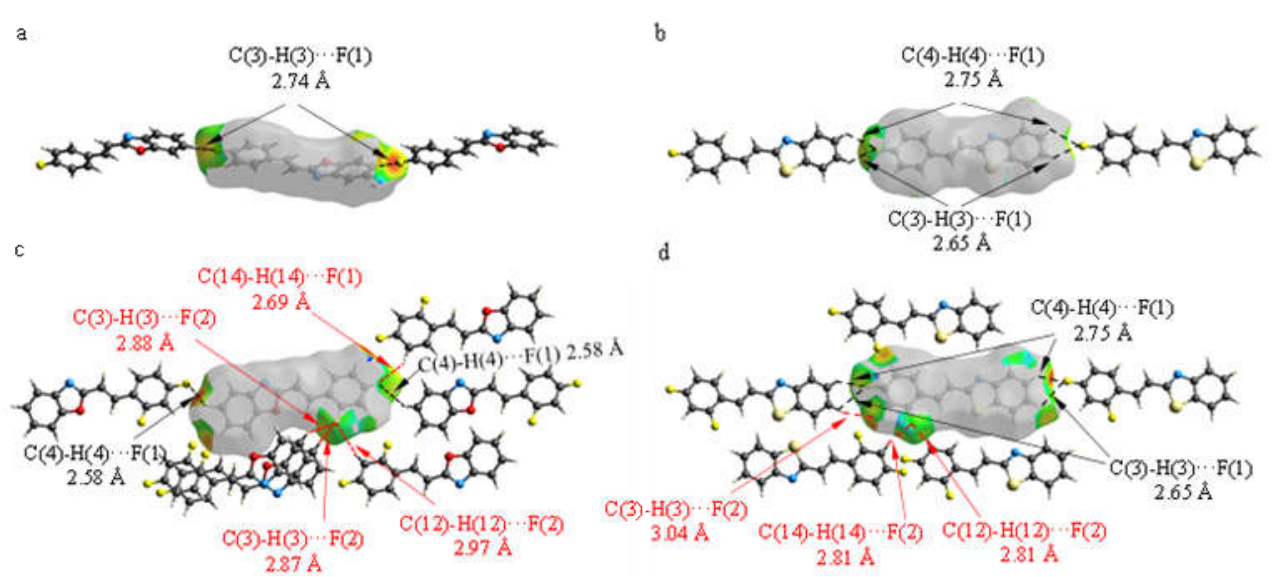

Figure 5 The Hirshfeld surfaces (mapped over $d_{i}$ ) and the distances of F $\cdots \cdot$-C interaction of (a) BOAF4, (b) BOAF24, (c) BTAF4 and(d) BOAF24.

For investigating the different molecular packing within crystals, Hirshfeld surface and two dimensional fingerprint (Supporting Information Figure S12) plots of crystal stacking based on $\mathrm{F} \cdots \mathrm{H}-\mathrm{C}$ interaction in crystals ofBOAF4, BOAF24, BTAF4 and BOAF24 were firstly calculated. For BOAF4, there was only one kind of $\mathrm{H}$-bond of $\mathrm{C}(3)-\mathrm{H}(3) \cdots \mathrm{F}(1)$ with a distance of $2.74 \AA$, and the $\mathrm{F} \cdots \mathrm{H}-\mathrm{C}$ interaction (the colored part in Figure $5 \mathrm{a}$ ) 
makes the molecule align along the long axis of the crystal. However, except for the similar $\mathrm{H}$-bond of $C(4)-H(4) \cdots F(1)(2.69 \AA)$, four additional H-bonds of $C(14)-H(14) \cdots F(1)(2.69 \AA), C(3)-H(3) \cdots F(2)(2.88 \AA)$, $C(3)-H(3) \cdots F(2)(2.87 \AA)$ and $C(12)-H(12) \cdots F(2)(2.97 \AA)$ were formed in the crystal of BOAF24. Besides, the

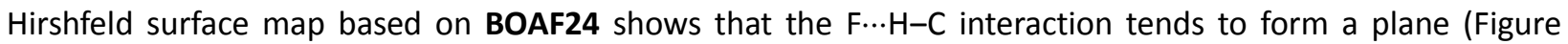
5c).Thus, the multiple $\mathrm{H}$-bonds induced the molecules to align perpendicularly to the long axis of the crystal. Furthermore, $\mathrm{F} \cdots \mathrm{H}-\mathrm{C}$ interactions in BOAF24 account for $23.0 \%$ of the total intermolecular interactions, which is twice as much as that of BOAF4 (12.2\%) (Table S8). Therefore, we believe that the F $\cdots \mathrm{H}-\mathrm{C}$ interaction is the main driving force for the different molecular packing in the crystals. The analysis of $\mathrm{F} \cdots \mathrm{H}-\mathrm{C}$ interactions in the single crystals of BTAF4 and BTAF24 was similar to those of BOAF4 and BOAF24 (Figure 5b and 5d).

To explore the mechanical property of the crystal, theoretical calculation was carried out with the density functional theory using the VASP software. The elastic properties were conducted by imposing small strains along the specific direction to obtain the stiffness tensor. As listed in Table S9, the obtained Pugh ratio for the crystalofBOAF4, BOAF24, BTAF4 and BTAF24 was 2.7280, 2.1115, 2.2372 and 1.9556, respectively. All of the values are larger than 1.75 , indicating that they are elastic. ${ }^{52}$ Moreover, it was observed that the Young's modulus of the phototropic surfaces of crystal BOAF4, BOAF24, BTAF4 and BTAF24 were 3.06 GPa, 7.32 GPa, 6.02 GPa and 4.05 GPa, respectively (Supporting Information Figure S13-S16). Thus, all the crystals are relatively easy to bend on the phototropic surface.

\section{Applications}

To demonstrate the intrinsic potential of these photoactuators, a pushing experiment was performed on glass substrate. Considering the high Young's modulus, BOAF24 crystal was chosen as a representative. As shown in Supporting Information Figure S17, a piece of cotton was adhered to the end of the rod-like crystal of BOAF24. Following photoirradiation, the crystal bent away from the light source with the cotton for about $0.2 \mathrm{~mm}$, indicating that the crystal had sufficient actuation power to push objects. To further evaluate the capability of 
the actuator, a much heavier organic crystal was employed. As presented in Figure 6a and Supporting Information Video S12, the organic crystal was pushed away from its original position at about $0.3 \mathrm{~mm}$ under UV irradiation. Thus, it is suggested that BOAF24 crystal, as a photoactuator, can perform mechanical work.
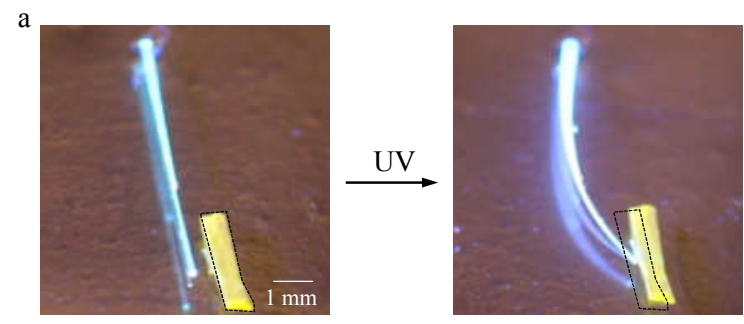

$\mathrm{b}$
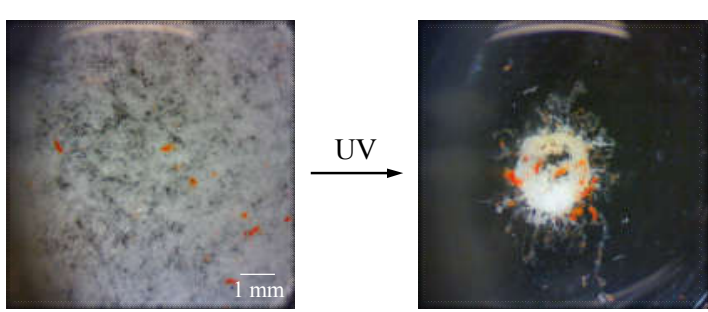

Figure 6 (a)Movement of a yellow organic crystal by the rod-like crystal of BOAF24 on UV irradiation (the dotted lines depict the edge of the crystal before irradiation).(b) The nanofibers of BOAF24 shrink and enrich the pollutants under the UV irradiation.

Except for pushing weights, fibers forming of BOAF24 in aqueous medium can even grab the undissolved objects. Here, an organic compound was utilized for the demonstration. As shown in Figure $6 \mathrm{~b}$ and Supporting Information Video S13, upon UV irradiation, BOAF24 fibers began to shrink and then assemble into a small ball. Meanwhile, it gradually seized the organic compound dispersed in water and finally collected them into the small ball. A possible working mechanism is proposed below. In water, the fibers arranged very loosely and intertwined with each other due to the poor solubility. Then [2+2] cycloaddition took place upon UV irradiation, which made the fibers firstly bend and curve, then knot and tear, and finally assemble into the small ball. Therefore, besides working as a conventional actuator, BOAF24 is endowed with the potential application in removal of the undissolved pollutants from water. 


\section{Conclusion}

In summary, styrylbenzoxazoles-based (BOAF4 and BOAF24) and styrylbenzothiazoles-based (BTAF4 and BTAF24) molecular crystals exhibited photosalient properties. Unexpectedly, BOAF4 and BTAF4 crystals exhibited bending behavior towards UV light, whereas BOAF24 and BTAF24 crystals showed negative phototropism behavior. This interesting phenomenon is due to the different molecular packing resulting from different $\mathrm{F} \cdots \mathrm{H}-\mathrm{C}$ interactions in the crystals. In detail, the F $\cdots \mathrm{H}-\mathrm{C}$ interactions of BOAF4 and BTAF4 crystals are linearly extensive, whereas the F $\cdots H-C$ interaction of BOAF24 and BTAF24 crystals has multiple directions. The different bending behaviors resulting from minor changes in molecular structure give us a new inspiration to design new photosalient crystals. It is the first report on exploring the relationship between the bending behavior and the molecular packing in organic crystals caused by [2+2] cycloaddition. Moreover, we obtained the physical properties of the crystals by theoretical calculation and found that they are elastic, which is a simple way to evaluate the mechanical properties of crystals. Taking advantages of the photo responsive property, we achieved the potential application in pushing objects, as well as enriching and removing pollutants. Herein, we proposed and achieved varying photo responsive behaviors of elastic crystals by molecular design, which opens a novel gate for crystal engineering.

\section{Supporting Information}

Supporting Information is available.

\section{Conflict of Interest}

There is no conflict of interest to report.

\section{Acknowledgements}


We are grateful for financial support from the National Science Foundation of China $(51773067,21788102)$, the Research Grants of Council of Hong Kong (C6009-17G), the Innovation of Technology Commission (ITCCNERC14SC01) and the National Key Research and Development Program of China (2018YFE0190200).

\section{References}

1. Sakai, T.; Kagawa, T.; Kasahara, M.; Swartz, T. E.; Christie, J. M.; Briggs, W. R.; Wada, M.; Okada, K. Nph1 and npl1: Blue light receptors that mediate both phototropism and chloroplast relocation. Proc. Natl. Acad. Sci. U. S. A., $2001,98,6969-6974$

2. Atamian, H. S.; Creux, N. M.; Brown, E. A.; Garner, A. G.; Blackman, B. K.; Harmer, S. L. Circadian regulation of sunflower heliotropism, floral orientation, and pollinator visits. Science, 2016, 353, 587-590

3. Dietrich, D.; Pang, L.; Kobayashi, A.; Fozard, J. A.; Boudolf, V.; Bhosale, R.; Antoni, R.; Nguyen, T.; Hiratsuka, S.; Fujii, N.; Miyazawa, Y.; Bae, T.-W.; Wells, D. M.; Owen, M. R.; Band, L. R.; Dyson, R. J.; Jensen, O. E.; King, J. R.; Tracy, S. R.; Sturrock, C. J.; Mooney, S. J.; Roberts, J. A.; Bhalerao, R. P.; Dinneny, J. R.; Rodriguez, P. L.; Nagatani, A.; Hosokawa, Y.; Baskin, T. I.; Pridmore, T. P.; De Veylder, L.; Takahashi, H.; Bennett, M. J. Root hydrotropism is controlled via a cortex-specific growth mechanism. Nat. Plants, 2017, 3, 17057

4. Medishetty, R.; Husain, A.; Bai, Z.; Runcevski, T.; Dinnebier, R. E.; Naumov, P.; Vittal, J. J. Single crystals popping under uv light: A photosalient effect triggered by a [2+2] cycloaddition reaction. Angew. Chem. Int. Ed., 2014, 53, 5907-5911

5. Nath, N. K.; Runcevski, T.; Lai, C. Y.; Chiesa, M.; Dinnebier, R. E.; Naumov, P. Surface and bulk effects in photochemical reactions and photomechanical effects in dynamic molecular crystals. J. Am. Chem. Soc., 2015, $137,13866-13875$

6. Naumov, P.; Chizhik, S.; Panda, M. K.; Nath, N. K.; Boldyreva, E. Mechanically responsive molecular crystals. Chem. Rev., 2015, 115, 12440-12490 
7. Zhang, L.; Naumov, P. Light- and humidity-induced motion of an acidochromic film. Angew. Chem. Int. Ed., $2015,54,8642-8647$

8. Wani, O. M.; Zeng, H.; Priimagi, A. A light-driven artificial flytrap. Nat. Commun., 2017, 8, 15546

9. Vale, R. D.; Milligan, R. A. The way things move: Looking under the hood of molecular motor proteins. Science, 2000, 288, 88-95

10. Chen, J.; Leung, F. K.; Stuart, M. C. A.; Kajitani, T.; Fukushima, T.; van der Giessen, E.; Feringa, B. L. Artificial muscle-like function from hierarchical supramolecular assembly of photoresponsive molecular motors. Nat. Chem., 2018, 10, 132-138

11. Wie, J. J.; Shankar, M. R.; White, T. J. Photomotility of polymers. Nat. Commun., 2016, 7, 13260

12. Ma, Z. C.; Zhang, Y. L.; Han, B.; Liu, X. Q.; Zhang, H. Z.; Chen, Q. D.; Sun, H. B. Femtosecond laser direct writing of plasmonic ag/pd alloy nanostructures enables flexible integration of robust sers substrates. $A d v$. Mater. Technol., 2017, 2, 1600270

13. Kobatake, S.; Takami, S.; Muto, H.; Ishikawa, T.; Irie, M. Rapid and reversible shape changes of molecular crystals on photoirradiation. Nature, 2007, 446, 778

14. Panda, M. K.; Ghosh, S.; Yasuda, N.; Moriwaki, T.; Mukherjee, G. D.; Reddy, C. M.; Naumov, P. Spatially resolved analysis of short-range structure perturbations in a plastically bent molecular crystal. Nat. Chem., 2015, 7, 65-72

15. Kim, T.; Al-Muhanna, M. K.; Al-Suwaidan, S. D.; Al-Kaysi, R. O.; Bardeen, C. J. Photoinduced curling of organic molecular crystal nanowires. Angew. Chem. Int. Ed., 2013, 52, 6889-6893 
16. Koshima, H.; Matsuo, R.; Matsudomi, M.; Uemura, Y.; Shiro, M. Light-driven bending crystals of salicylidenephenylethylamines in enantiomeric and racemate forms. Cryst. Growth Des., 2013, 13, 4330-4337

17. Bushuyev, O. S.; Tomberg, A.; Friscic, T.; Barrett, C. J. Shaping crystals with light: Crystal-to-crystal isomerization and photomechanical effect in fluorinated azobenzenes. J. Am. Chem. Soc., 2013, 135, 1255612559

18. Nath, N. K.; Pejov, L.; Nichols, S. M.; Hu, C.; Saleh, N. i.; Kahr, B.; Naumov, P. Model for photoinduced bending of slender molecular crystals. J. Am. Chem. Soc., 2014, 136, 2757-2766

19. Ohshima, S.; Morimoto, M.; Irie, M. Light-driven bending of diarylethene mixed crystals. Chem. Sci., 2015, $6,5746-5752$

20. Kitagawa, D.; Tanaka, R.; Kobatake, S. Photoinduced stepwise bending behavior of photochromic diarylethene crystals. Crystengcomm, 2016, 18, 7236-7240

21. Koshima, H.; Uchimoto, H.; Taniguchi, T.; Nakamura, J.; Asahi, T.; Asahi, T. Mechanical motion of molecular crystals induced by [4+4] photodimerisation. Crystengcomm, 2016, 18, 7305-7310

22. Al-Kaysi, R. O.; Tong, F.; Al-Haidar, M.; Zhu, L.; Bardeen, C. J. Highly branched photomechanical crystals. Chem. Commun., 2017, 53, 2622-2625

23. Hirano, A.; Hashimoto, T.; Kitagawa, D.; Kono, K.; Kobatake, S. Dependence of photoinduced bending behavior of diarylethene crystals on ultraviolet irradiation power. Cryst. Growth Des., 2017, 17, 4819-4825

24. Kitagawa, D.; Kawasaki, K.; Tanaka, R.; Kobatake, S. Mechanical behavior of molecular crystals induced by combination of photochromic reaction and reversible single-crystal-to-single crystal phase transition. Chem. Mater., 2017, 29, 7524-7532 
25. Cheng, S.-C.; Chen, K.-J.; Suzaki, Y.; Tsuchido, Y.; Kuo, T.-S.; Osakada, K.; Horie, M. Reversible laser-induced bending of pseudorotaxane crystals. J. Am. Chem. Soc., 2018, 140, 90-93

26. Samanta, R.; Ghosh, S.; Devarapalli, R.; Reddy, C. M. Visible light mediated photopolymerization in single crystals: Photomechanical bending and thermomechanical unbending. Chem. Mater., 2018, 30, 577-581

27. Liu, J.; Ye, K.; Shen, Y.; Peng, J.; Sun, J.; Lu, R. Photoactuators based on the dynamic molecularcrystals of naphthalene acrylic acids driven by stereospecific [2+2] cycloaddition reactions. J. Mater. Chem. C, 2020, 8, $3165-3175$

28. Peng, J.; Ye, K.; Liu, C.; Sun, J.; Lu, R. The photomechanic effects of the molecular crystals based on 5chloro-2-(naphthalenylvinyl)benzoxazols fueled by topo-photochemical reactions. J. Mater. Chem. C, 2019, 7, 5433-5441

29. Peng, J.; Ye, K.; Yue, Y.; Liu, C.; Sun, J.; Lu, R. Photomechanical effects of acidochromic diarylethene derivatives. Sci. Sin. Chim., 2020, 50, 108-117

30. Uchida, K.; Sukata, S.; Matsuzawa, Y.; Akazawa, M.; de Jong, J. J.; Katsonis, N.; Kojima, Y.; Nakamura, S.; Areephong, J.; Meetsma, A.; Feringa, B. L. Photoresponsive rolling and bending of thin crystals of chiral diarylethenes. Chem. Commun., 2008, 100, 326-328

31. Shtukenberg, A. G.; Freudenthal, J.; Kahr, B. Reversible twisting during helical hippuric acid crystal growth. J. Am. Chem. Soc., 2010, 132, 9341-9349

32. Kitagawa, D.; Nishi, H.; Kobatake, S. Photoinduced twisting of a photochromic diarylethene crystal. Angew. Chem. Int. Ed., 2013, 52, 9320-9322 
33. Natarajan, A.; Tsai, C. K.; Khan, S. I.; Mccarren, P.; Houk, K. N.; Garcia-Garibay, M. A. The photoarrangement of alpha-santonin is a single-crystal-to-single-crystal reaction: A long kept secret in solidstate organic chemistry revealed. J. Am. Chem. Soc., 2007, 129, 9846-9847

34. Commins, P.; Natarajan, A.; Tsai, C. K.; Khan, S. I.; Nath, N. K.; Naumov, P.; Garciagaribay, M. A. Structurereactivity correlations and mechanistic understanding of the photorearrangement and photosalient effect of $\alpha$-santonin and its derivatives in solutions, crystals, and nanocrystalline suspensions. Cryst. Growth Des., 2015, $15,1983-1990$

35. Ghosh, S.; Mishra, M. K.; Ganguly, S.; Desiraju, G. R. Dual stress and thermally driven mechanical properties of the same organic crystal: 2,6-dichlorobenzylidene-4-fluoro-3-nitroaniline. J. Am. Chem. Soc., 2015, 137, 9912-9921

36. Medishetty, R.; Sahoo, S. C.; Mulijanto, C. E.; Naumov, P.; Vittal, J. J. Photosalient behavior of photoreactive crystals. Chem. Mater., 2015, 27, 1821-1829

37. Uchida, E.; Azumi, R.; Norikane, Y. Light-induced crawling of crystals on a glass surface. Nat. Commun., $2015,6,7310$

38. Gupta, P.; Panda, T.; Allu, S.; Borah, S.; Baishya, A.; Gunnam, A.; Nangia, A.; Naumov, P.; Nath, N. K. Crystalline acylhydrazone photoswitches with multiple mechanical responses. Cryst. Growth Des., 2019, 19, 3039-3044

39. Gupta, P.; Karothu, D. P.; Ahmed, E.; Naumov, P.; Nath, N. K. Thermally twistable, photobendable, elastically deformable, and self-healable soft crystals. Angew. Chem. Int. Ed., 2018, 57, 8498-8502

40. Kitagawa, D.; Tsujioka, H.; Tong, F.; Dong, X.; Bardeen, C. J.; Kobatake, S. Control of photomechanical crystal twisting by illumination direction. J. Am. Chem. Soc., 2018, 140, 4208-4212 
41. Wang, H.; Chen, P.; Wu, Z.; Zhao, J.; Sun, J.; Lu, R. Bending, curling, rolling, and salient behavior of molecular crystals driven by [2+2] cycloaddition of a styrylbenzoxazole derivative. Angew. Chem. Int. Ed., 2017, 56, 9463-9467

42. Wang, H.; Zhao, J.; Yang, G.; Zhang, F.; Sun, J.; Lu, R. Diarylethene-based xerogels: the fabrication of more entangled networks driven by isomerization and acidofluorochromism. Org. Biomol. Chem., 2018, 16, 21142124

43. Liu, Z.; Zhou, C.; Lei, T.; Nan, X.-L.; Chen, B.; Tung, C.-H.; Wu, L.-Z. Aggregation-enabled intermolecular photo[2+2]cycloaddition of aryl terminal olefins by visible-light catalysis. CCS Chem., 2020, 2, 582-588

44. Li, J.; Shen, P.; Zhao, Z.; Tang, B. Z. Through-space conjugation: A thriving alternative for optoelectronic materials. CCS Chem., 2019, 1, 181-196

45. Wei, P.; Zhang, J.-X.; Zhao, Z.; Chen, Y.; He, X.; Chen, M.; Gong, J.; Sung, H. H. Y.; Williams, I. D.; Lam, J. W. Y.; Tang, B. Z. Multiple yet controllable photoswitching in a single aiegen system. J. Am. Chem. Soc., 2018, 140, 1966-1975

46. Wang, H.; Xing, H.; Gong, J.; Zhang, H.; Zhang, J.; Wei, P.; Yang, G.; Lam, J. W. Y.; Lu, R.; Tang, B. Z. "Living” luminogens: Light driven acq-to-aie transformation accompanied with solid-state actuation. Mater. Horiz., 2020, doi.org/10.1039/D1030MH00447B

47. Schmidt, G. Photodimerization in the solid state. Pure Appl. Chem., 1971, 27, 647-678

48. Ikeda, T.; Nakano, M.; Yu, Y.; Tsutsumi, O.; Kanazawa, A. Anisotropic bending and unbending behavior of azobenzene liquid-crystalline gels by light exposure. Adv. Mater., 2003, 15, 201-205

49. Yu, Y.; Nakano, M.; Ikeda, T. Directed bending of a polymer film by light. Nature, $\mathbf{2 0 0 3 , 4 2 5 , ~ 1 4 5 - 1 4 5}$ 
50. Kondo, M.; Yu, Y.; Ikeda, T. How does the initial alignment of mesogens affect the photoinduced bending behavior of liquid-crystalline elastomers? Angew. Chem. Int. Ed., 2006, 45, 1378-1382

51. Sun, J.-K.; Li, W.; Chen, C.; Ren, C.-X.; Pan, D.-M.; Zhang, J. Photoinduced bending of a large single crystal of a 1,2-bis(4-pyridyl)ethylene-based pyridinium salt powered by a [2+2] cycloaddition. Angew. Chem. Int. Ed., 2013, 52, 6653-6657

52. Pugh, S. F. Xcii. Relations between the elastic moduli and the plastic properties of polycrystalline pure metals. The London, Edinburgh, and Dublin Philosophical Magazine and Journal of Science, 1954, 45, 823-843 